\title{
Limit Gibbs State for Some Classes of One-Dimensional Systems of Quantum Statistical Mechanics ${ }^{\star}$
}

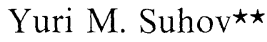 \\ Centre de Physique Theorique, CNRS, F-13274 Marseille, Cedex 2, and I.H.E.S., F-91440 Bures-sur- \\ Yvette, France and Istituto Matematico, Universita di Camerino, I-Camerino MC, Italy
}

\begin{abstract}
We prove the existence of the limit Gibbs state for one-dimensional continuous quantum fermion systems with non-hard-core, non-negative, rapidly decreasing pair interaction potentials. Existence of the limit Gibbs state is also established for one-dimensional continuous quantum boson systems with pair interaction potentials as above which, in addition, increase sufficiently fast at small distances.
\end{abstract}

\section{Introduction}

The mathematically rigorous theory of phase transitions in systems with infinitely many degrees of freedom [1-3] is now developped in the main for the class of spin systems (see, e.g., $[4,5]$ and references there). The case of continuous systems, particularly, of continuous quantum systems (c.q.s.) is more difficult. Even fundamental physical notions such as those of time evolution and equilibrium states have yet no satisfactory definitions, except a number of exactly solvable models $[3,6]$. One of principal difficulties is that the Hamiltonians of c.q.s. in bounded domains are unbounded operators.

The main method for studying the c.q.s. is now the Wiener integral representation based on the Feynman-Kac formula and used first by Ginibre [7]. This method allows one to exploit an analogy between classical and quantum systems and to investigate dilute quantum gases [7] and one-dimensional c.q.s. [8]. In particular, in [8] we considered the case of one-dimensional c.q.s. with a hardcore, long-range interaction between particles.

The present paper deals with several types of one-dimensional c.q.s. where the particles interact via a non-hard-core long-range interaction potential which is non-negative and rapidly decreasing. We establish here the existence of the limit Gibbs state in the infinite volume. Its properties such as regularity (cluster

* This work is partially performed with the support of the National Council of Researches of Italy $\star \star$ Permanent address: Institute for Problems of Information Transmission, USSR Academy of Sciences, 19, av. Yermolovoj, 103051 Moscow, USSR 
property), etc., which illustrate the absence of phase transitions will be proved in a separate paper.

Notice that c.q.s. have, in general, some properties which are surprising from the point of view of a "traditional folklore". In particular, even such model as the one-dimensional free boson c.q.s. may exhibit a phase transition [9]. This suggests the detailed study of "simple" classes of c.q.s. where the physical arguments lead to the hypothesis about the absence of phase transitions.

The method of the paper is analogous to that used earlier in the case of onedimensional classical systems [10]. The main step in the proof is a transfer matrix formalism (see Section 8) combined with some preliminary estimations (Sections 5-7).

In the Appendix we briefly sketch generalizations of the results to some other classes of one-dimensional c.q.s.

In the second part of the introduction, let us fix the interaction of particles we consider. Denote $R_{+}^{1}=(0, \infty)$ and $\bar{R}_{+}^{1}=[0, \infty)$. Suppose $\Phi: R_{+}^{1} \rightarrow \bar{R}_{+}^{1}$ is a $C^{0}$-function, possibly having a singularity at the origin ${ }^{1}$.

We shall impose the following conditions:

a) there exist $d \geqq 0$ and a monotonic function $\psi: R_{+}^{1} \rightarrow \bar{R}_{+}^{1}$ such that $\lim _{r \rightarrow \infty} e^{\alpha r} \psi(r)=0$ for every $\alpha>0$, and $\Phi(r) \leqq \psi(r)$ provided $r>d$,

b) there exist $d^{\sim}>0, a>1$ and a monotonic function $\psi^{\sim}: R_{+}^{1} \rightarrow \bar{R}_{+}^{1}$ such that $d^{2}$

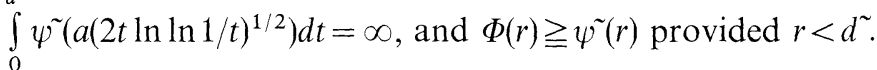

Condition $b$ ) is used only for the boson case. The more strong condition is sufficient for b): for some $\varepsilon>0$ and $\tilde{d_{1}}>0$

$$
\int_{0}^{d_{1}} \psi^{\tilde{Y}(t) t^{1+\varepsilon}} d t=+\infty
$$

Let $\Lambda$ be a bounded interval of $R^{1}$. As usually, we consider the $n$-particle Schrödinger operators $\hat{H}_{n}(\Lambda)$ in $L^{2}\left(\Lambda^{n}\right)$ :

$$
\left\{\begin{array}{c}
\hat{H}_{0}(\Lambda)=0, \quad \hat{H}_{1}(\Lambda)=-1 / 2 d^{2} / d x^{2}, \\
\hat{H}_{n}(\Lambda)=-1 / 2 \sum_{i=1}^{n} \partial^{2} / \partial x_{i}^{2}+\sum_{1 \leqq i<j \leqq n} \Phi\left(\left|x_{i}-x_{j}\right|\right), \quad n=2,3, \ldots .
\end{array}\right.
$$

They are symmetric on natural domains in $L^{2}\left(\Lambda^{n}\right)$. We shall deal with the selfadjoint extension $H_{n}(\Lambda)$ of $\hat{H}_{n}(\Lambda)$ which is given as follows. Consider the semigroup of operators in $L^{2}\left(\Lambda^{n}\right)$ given by the kernels

$$
\begin{aligned}
W_{t}\left(\bar{x}^{n}, \bar{y}^{n}\right)= & \int d P_{\bar{x}^{n}, \bar{y}^{n}}^{t}\left(\bar{\omega}^{n}\right) \alpha_{\Lambda}\left(\bar{\omega}^{n}\right) \\
& \cdot \exp \left[-U\left(\bar{\omega}^{n}\right)\right], \bar{x}^{n}, \bar{y}^{n} \in \Lambda^{n}, t>0,
\end{aligned}
$$

see $[7,(2.7)]$ whence the notations are taken. We take, as $H_{n}(\Lambda)$, the generator of this semigroup. It corresponds to the Dirichlet boundary conditions on $\partial \Lambda^{n}{ }^{2}$

1 We exclude the case of non-zero hard cores considered in [8]

2 The extension of the results to other types of boundary conditions on $\partial \Lambda^{n}$ will be given in the second part of the work 
and, if $\Phi$ is "too singular" at the origin, on the diagonal part of $\Lambda^{n}$ [as we shall see, it is the case if $\Phi$ satisfies condition b) above]. See [7, Section 1.3] for connections between $(0.1)$ and $(0.2)$.

\section{Gibbs State in a Finite Volume}

We adopt in the main the definitions and notations of [1, Chapter 1, 3, 5-7]. Let $\Omega \subset R^{1}$ be a bounded Borel set (b.B.s.), $\mathscr{H}_{\varepsilon}(\Omega)=\bigoplus_{n=0}^{\infty} L_{\varepsilon}^{2}\left(\Lambda^{n}\right)$ be the Fock space of a boson $(\varepsilon=+)$ or fermion $(\varepsilon=-)$ c.q.s. in $\Omega, \mathfrak{U}_{\Omega}^{\varepsilon}$ be the $C^{*}$-algebra of bounded operators in $\mathscr{H}_{\varepsilon}(\Omega)$ and $\mathfrak{U}^{\varepsilon}$ be the $B^{*}$-algebra of the infinite boson or fermion c.q.s. in $R^{1}: \mathfrak{U}^{\varepsilon}$ is the completition (in norm) of the inductive limit (or union, if one prefers) $\tilde{\mathfrak{U}}^{\varepsilon}$ of $\mathfrak{U}_{\Omega}^{\varepsilon}$. Let $\left\{\tau_{a}, a \in R^{1}\right\}$ be the group of space translations acting on $\mathfrak{A}^{\varepsilon}$.

Given a bounded interval $\Lambda \subset R^{1}$, we denote $H_{\varepsilon, n}(\Lambda)=P_{\varepsilon, n} H_{n}(\Lambda) P_{\varepsilon, n}$ where the operator $H_{n}(\Lambda)$ is introducted in the preceding section, and $P_{\varepsilon, n}: L^{2}\left(\Lambda^{n}\right) \rightarrow L_{\varepsilon}^{2}\left(\Lambda^{n}\right)$ is the "canonical" projection. Let $\beta \in R_{+}^{1}$ and $\mu \in R^{1}$; consider the positive operator $E_{\beta, \mu}^{\varepsilon}(\Lambda)=\bigoplus_{n=0}^{\infty} e^{\beta \mu n} \exp \left[-\beta H_{\varepsilon, n}(\Lambda)\right]$ in $\mathscr{H}_{\varepsilon}(\Lambda)$. If

$$
\Xi_{\beta, \mu, \Lambda}^{\varepsilon}=\sum_{n=0}^{\infty} e^{\beta \mu n} \operatorname{tr}_{L_{\varepsilon}^{2}\left(\Lambda^{n}\right)} \exp \left[-\beta H_{\varepsilon, n}(\Lambda)\right]<\infty,
$$

then $E_{\beta, \mu}^{\varepsilon}(\Lambda)$ is of trace class, and $\Xi_{\beta, \mu, \Lambda}^{\varepsilon}=\operatorname{tr}_{\mathscr{H}_{\varepsilon}(\Lambda)} E_{\beta, \mu}^{\varepsilon}(\Lambda)$.

The Gibbs state of the c.q.s. in $\Lambda$ is given by

$$
G_{\beta, \mu, \Lambda}^{\varepsilon}(A)=\operatorname{tr}_{\mathscr{H}_{\varepsilon}(A)} g_{\beta, \mu, \Lambda}^{\varepsilon} A, \quad A \in \mathfrak{U}_{\Lambda}^{\varepsilon},
$$

where $g_{\beta, \mu, \Lambda}^{\varepsilon}=\left(\Xi_{\beta, \mu, \Lambda}^{\varepsilon}\right)^{-1} E_{\beta, \mu}^{\varepsilon},(\Lambda)$.

Proposition 1.1. Let $\Phi$ satisfy conditions a) and b) above for $\varepsilon=+$ and condition a) above for $\varepsilon=-$. Then for any $\beta \in R_{+}^{1}$ and $\mu \in R^{1}, \Xi_{\beta, \mu, A}^{\varepsilon}<\infty$ and the RHS of (1.1) defines a state of $\mathfrak{U}_{\Lambda}^{\varepsilon}$.

The proof of Proposition 1.1 is carried out in Section 3. It is convenient to consider $G_{\beta, \mu, \Lambda}^{\varepsilon}$ as the state of the $C^{*}$-algebra $\mathfrak{A}^{\varepsilon}$ using the "canonical" inclusion $\mathfrak{U}_{\Lambda}^{\varepsilon} \rightarrow \mathfrak{U}^{\varepsilon}$. If $\Omega \subset \Lambda$, then the restriction of $G_{\beta, \mu, \Lambda}^{\varepsilon}$ to $\mathfrak{U}_{\Omega}^{\varepsilon}$ is given by a positive trace class operator $g_{\beta, \mu, \Lambda}^{\varepsilon, \Omega}$ in $\mathscr{H}_{\varepsilon}(\Omega)$ with $\operatorname{tr}_{\mathscr{H}_{\varepsilon}(\Omega)} g_{\beta, \mu, \Lambda}^{\varepsilon, \Omega}=1$ :

$$
G_{\beta, \mu, A}^{\varepsilon}(A)=\operatorname{tr}_{\mathscr{H}_{\varepsilon}(\Omega)} g_{\beta, \mu, \Lambda}^{\varepsilon, \Omega} A, \quad A \in \mathfrak{O}_{\Omega}^{\varepsilon} .
$$

It is not hard to check that, with the "canonical" identification $\mathscr{H}_{\varepsilon}(\Lambda)$

$=\mathscr{H}_{\varepsilon}(\Omega) \otimes \mathscr{H}_{\varepsilon}(\Lambda \backslash \Omega)($ see $[1$, Section 7.1]),

$$
g_{\beta, \mu, \Lambda}^{\varepsilon, \Omega}=\operatorname{tr}_{\mathscr{H}_{\varepsilon}(\Lambda \backslash \Omega)} g_{\beta, \mu, \Lambda}^{\varepsilon} .
$$

The operator $g_{\beta, \mu, A}^{\varepsilon, \Omega}$ being of trace class is given by its kernel $\varrho_{\beta, \mu, A}^{\varepsilon, \Omega}$ : $\left[\bigcup_{n=0}^{\infty} \Omega^{n}\right] \times\left[\bigcup_{m=0}^{\infty} \Omega^{m}\right] \rightarrow C^{1}\left[\Omega^{0}\right.$ consists of the unique element (vacuum) $]$. Namely, if $f=\bigoplus_{n=0}^{\infty} f_{n}, f_{n} \in L_{\varepsilon}^{2}\left(\Omega^{n}\right)$, then $g_{\beta, \mu, A}^{\varepsilon, \Omega} f=\bigoplus_{n=0}^{\infty} \tilde{f}_{n}$, where

$$
\tilde{f}_{n}\left(\bar{x}^{n}\right)=\sum_{m=0}^{\infty} \int_{\Omega^{m}} d \bar{y}^{m} \varrho_{\beta, \mu, \Lambda}^{\varepsilon, \Omega} \cdot\left(\bar{x}^{n}, \bar{y}^{m}\right) f_{m}\left(\bar{y}^{m}\right), \quad \bar{x}^{n} \in \Omega^{n}, \quad n=0,1, \ldots
$$


Actually, in our case, $\varrho_{\beta, \mu, \Lambda}^{\varepsilon, \Omega}\left(\bar{x}^{n}, \bar{y}^{m}\right)$ takes only real values and vanishes for $m \neq n$ (see [7, Sections 1.3 and 2.1]). Hence, we deal with a function $\varrho_{\beta, \mu, \Lambda}^{\varepsilon, \Omega}: \bigcup_{n=0}^{\infty}\left(\Omega^{n} \times \Omega^{n}\right) \rightarrow R^{1}$

\section{Results}

In what follows we always suppose that $\Phi$ satisfies the conditions of Proposition 1.1 and do not specify this every time again. Let $A \rightarrow R^{1}$ denote the set of finite intervals $A \subset R^{1}$ directed by inclusion. The main results of the present work are the two following theorems:

Theorem 1. Let $A \in \mathfrak{U}^{\varepsilon}$. For any $\beta \in R_{+}^{1}$ and $\mu \in R^{1}$ there exists the limit

$$
G_{\beta, \mu}^{\varepsilon}(A)=\lim _{\Lambda \rightarrow R^{1}} G_{\beta, \mu, A}^{\varepsilon}(A) .
$$

The limit state $G_{\beta, \mu}^{\varepsilon}: \mathfrak{U}^{\varepsilon} \rightarrow C^{1}$ is locally normal and invariant under action of the group $\left\{\tau_{a}, a \in R^{1}\right\}$.

Theorem 2. The states $G_{\beta, \mu}^{\varepsilon}$ are extremal points of the set of locally normal states invariant under action of $\left\{\tau_{a}, a \in R^{1}\right\}$.

In this section we give a sketch of the proof of the both theorems. The first (and main) step in the proof of Theorem 1 is

Theorem 3. For any b.B.s. $\Omega \subset R^{1}$ the operators $g_{\beta, \mu, \Lambda}^{\varepsilon, \Omega}$ converge to a limit $g_{\beta, \mu}^{\varepsilon, \Omega}$ as $\Lambda \rightarrow R^{1}$ in the Hilbert-Schmidt norm in $\mathscr{H}_{\varepsilon}(\Omega)$ :

$$
\text { HS- } \lim _{\Lambda \rightarrow R^{1}} g_{\beta, \mu, \Lambda}^{\varepsilon, \Omega}=g_{\beta, \mu}^{\varepsilon, \Omega} .
$$

The operator $g_{\beta, \mu}^{\varepsilon, \Omega}$ is non-negative and of trace class with $\operatorname{tr}_{\mathscr{H}}(\Omega) g_{\beta, \mu}^{\varepsilon, \Omega}=1$.

It follows from Theorem 3 and from Lemma 1 of [11] that the operators $g_{\beta, \mu, \Lambda}^{\varepsilon, \Omega}$ converge to the limit $g_{\beta, \mu}^{\varepsilon, \Omega}$ in the trace norm in $\mathscr{H}_{\varepsilon}(\Omega)$. Hence, for every $A \in \tilde{\mathfrak{A}}^{\varepsilon}$ there exists the limit (2.1), and for $A \in \mathfrak{U}_{\Omega}^{\varepsilon}, G_{\beta, \mu}^{\varepsilon}(A)=\operatorname{tr}_{\mathscr{H}_{\varepsilon}(\Omega)} g_{\beta, \mu}^{\varepsilon, \Omega} A$. This implies the convergence of $G_{\beta, \mu, \Lambda}^{\varepsilon}(A)$ for any $A \in \mathfrak{U}^{\varepsilon}$.

The invariance property of $G_{\beta, \mu}^{\varepsilon}$ follows from the relation

$$
\tau_{a} g_{\beta, \mu}^{\varepsilon, \Omega}=g_{\beta, \mu}^{\varepsilon, \Omega+a},
$$

where $\Omega+a=\left\{x \in R^{1}: x-a \in \Omega\right\}$ and $g_{\beta, \mu}^{\varepsilon, \Omega}, g_{\beta, \mu}^{\varepsilon, \Omega+a}$ are considered as elements of $\mathfrak{I}^{\varepsilon}$.

To prove Theorem 2, it suffices to verify that

$$
\lim _{|a| \rightarrow \infty} G_{\beta, \mu}^{\varepsilon}\left(A_{1} \tau_{a} A_{2}\right)=G_{\beta, \mu}^{\varepsilon}\left(A_{1}\right) G_{\beta, \mu}^{\varepsilon}\left(A_{2}\right), A_{1}, A_{2} \in \mathfrak{U}^{\varepsilon} ;
$$

in the case $\varepsilon=-$, we can consider $A_{1}, A_{2} \in \mathfrak{U}_{\text {even }}^{-}$[1, Sections 6.3 and 7.1]. Moreover, in both cases it is sufficient to take $A_{1}, A_{2} \in \tilde{\mathfrak{U}}^{\varepsilon}$. Let $\Omega_{1}, \Omega_{2} \subset R^{1}$ be b.B.s., and $a \in R^{1}$ be chosen so that $\Omega_{1} \cap\left(\Omega_{2}+a\right)=\emptyset$. Denote $\Omega(a)=\Omega_{1} \cup\left(\Omega_{2}+a\right)$. Let the operator $\tilde{g}_{\beta, u}^{\varepsilon, \Omega(a)}$ correspond to $g_{\beta, \mu}^{\varepsilon, \Omega(a)}$ by the identification $\mathscr{H}_{\varepsilon}(\Omega(a)) \simeq \mathscr{H}_{\varepsilon}\left(\Omega_{1}\right) \otimes \mathscr{H}_{\varepsilon}\left(\Omega_{2}\right)$. It is not hard to check that Equation (2.3) for $A_{i} \in \mathfrak{U}_{\Omega_{2}}^{\varepsilon}$ $\left(\mathfrak{U}_{\Omega_{l}, \text { even }}^{-}\right.$for $\left.\varepsilon=-\right), i=1,2$, follows from

$$
\lim _{|a| \rightarrow \infty}\left\|\tilde{g}_{\beta, \mu}^{\varepsilon, \Omega(a)}-g_{\beta, \mu}^{\varepsilon, \Omega_{1}} \otimes g_{\beta, \mu}^{\varepsilon, \Omega_{2}}\right\|_{\mathrm{tr}}=0,
$$


or, due to Lemma 1 of [11], from the corresponding Hilbert-Schmidt norm convergence.

Summarizing what is said above, we formulate

Theorem 4. i) For any $a \in R^{1}$ and b.B.s. $\Omega \subset R^{1}$ the operators $g_{\beta, \mu}^{\varepsilon, \Omega}$ obey (2.4).

ii) For any b.B.s. $\Omega_{1}$ and $\Omega_{2}$

$$
\lim _{|a| \rightarrow \infty}\left\|\tilde{g}_{\beta, \mu}^{\varepsilon, \Omega(a)}-g_{\beta, \mu}^{\varepsilon, \Omega_{1}} \otimes g_{\beta, \mu}^{\varepsilon, \Omega_{2}}\right\|_{\mathrm{HS}}=0 \text {. }
$$

In Sections 3-8 we prove Theorem 3. The proof of Theorem 4 will be completed in the next paper.

\section{The Ginibre Representation}

The analysis of $g_{\beta, \mu, \Lambda}^{\varepsilon, \Omega}$ is based on the representation of the kernels $\varrho_{\beta, \mu, \Lambda}^{\varepsilon, \Omega}$ in terms of Wiener integrals used by Ginibre $[7]^{3}$. We will use (with little changes) the notation system and definitions of [7]. For brevity, we omit, whenever it is possible, the indices $\beta, \mu$, and $\varepsilon$.

At first, let $\varepsilon=+$ (bosons). $\Xi_{A}^{+}$introduced in Section 1 has a general representation $[7,(2.33)]$. As we shall show, condition b) imposed on $\Phi$ allows us to simplify this representation and reduce, in a sense, the Bose statistics to the Maxwell-Boltzmann one.

Proposition 3.1. $\Xi_{A}^{+}$obeys the representation $[7,(2.16)]$ :

$$
\Xi_{\Lambda}^{+}=\int_{[\Lambda]} \mathrm{d} Y \alpha_{\Lambda}(\mathrm{Y}) \exp [-U(Y)+\beta n(Y)],
$$

where $[\Lambda]$ is the collection of the finite families of simple (time length $\beta$ ) closed loops starting in $\Lambda, n(Y)$ is the number of loops $\omega \in Y$ and the measure $d Y$ is given by $[(7,(2.12)-(2.13)]$.

Proof. Repeating the arguments of [7, pp. 358-361] which lead at [7, (2.31)(2.33)], one has to show that non-trivial permutations $\pi$ give the zero contribution in the final result $[7,(2.33)]$. In fact, the problem is to prove that, given $x_{1}, x_{2} \in R^{1}$, $x_{1} \neq x_{2}$, the equality $u\left(\omega_{1}, \omega_{2}\right)=\int_{0}^{\beta} \Phi\left(\left|\omega_{1}(t)-\omega_{2}(t)\right|\right) d t=\infty$ holds for a.a. pairs $\left(\omega_{1}, \omega_{2}\right)$ of the simple trajectories with $\omega_{1}(0)=\omega_{2}(\beta)=x_{1}, \omega_{1}(\beta)=\omega_{2}(0)=x_{2}$ w.r.t. the measure $d P_{x_{1}, x_{2}}^{\beta}\left(\omega_{1}\right) \times d P_{x_{2}, x_{1}}^{\beta}\left(\omega_{2}\right)$. Since $\omega(t)=\omega_{1}(t)-\omega_{2}(t)$ also forms the Wiener process, one has simply to show that $\int_{0}^{\beta} \Phi(|\omega(t)|) d t=\infty$ for a.a. $\omega$ w.r.t. the measure $d P_{-x, x}^{\beta}$.

Every trajectory $\omega$ with $\omega(0)=-x, \omega(\beta)=x$ passes through the origin (onedimensional case!). We use the fact that the (random) moment $t_{0}$ of the first passage through the origin is a Markov moment and the Wiener process is strictly markovian (see, e.g., [12, Chapter 1, Section 4]). As a consequence, we have that,

3 Formally speaking, singular potentials in one dimension are excluded from consideration in [7], see condition A) in [7, p. 346]. However, the formula $[7,(2.7)][(0.2)$ of Section 0$]$ and all others related to the Wiener integral structure (particularly, those leading at [7, (2.31)-(2.33)]) keep the sense for our case and are used without making such a reservation every time again 
given the value $t_{0} \in(0, \beta)$, the (conditional) probability distribution for the piece of the trajectory $\left(\omega(t) \mid t \in\left(t_{0}, \beta\right)\right)$ is merely given by the measure $d P_{0, x}^{\beta-t_{0}}$. Hence, the problem is reduced to the proof that $\int_{0}^{\beta-t_{0}} \Phi(|\omega(t)|) d t=\infty$ for a.a. $\omega$ w.r.t. the
measure $d P_{0}^{\beta-t_{0}}$.

Now we use the $\log \log$ low for the Wiener motion: for $\left(P_{0, x}^{\beta-t_{0}}\right)$-a.a. $\omega$, $\lim \sup (2 t \ln \ln 1 / t)^{-1 / 2}|\omega(t)|=1 \quad([13$, Chapter 6 , Section 4, Theorem 4]). Substituting $\psi^{\sim}$ instead of $\Phi$ and $a(2 t \ln \ln 1 / t)^{1 / 2}, a>1$, instead of $|\omega(t)|$, we arrive at condition $b$ ). This completes the proof.

Next pass to $\varepsilon=-$ (fermions). For this case we also modify formula [7, (2.33)]. Given $Y$, a finite family of simple closed loops, we set: $\alpha_{-}^{0}(Y)=0$ if there exists a pair $\omega, \omega^{\prime} \in Y, \omega \neq \omega^{\prime}$, with $\inf _{0 \leqq t \leqq \beta}\left|\omega(t)-\omega^{\prime}(t)\right|=0$, and $\alpha_{-}^{0}(Y)=1$, otherwise.

Proposition 3.2. $\Xi_{A}^{-}$obeys the following representation:

$$
\Xi_{\Lambda}^{-}=\int_{[\Lambda]} d Y \alpha_{\Lambda}(Y) \alpha_{-}^{0}(Y) \exp [-U(Y)+\beta \mu n(Y)],
$$

where, as above, $d Y$ is given by $[7,(2.12)$ and (2.13)].

Proof. Let $S_{n}(\Lambda)$ denote the $n$-dimensional symplex $\left\{\bar{x}^{n}=\left(x_{1}, \ldots, x_{n}\right) \in \Lambda^{n}: x_{1} \leqq \ldots\right.$ $\left.\leqq x_{n}\right\}$. The mapping $T_{n}: f \in L_{-}^{2}\left(\Lambda^{n}\right) !->n !\left(\left.f\right|_{S_{n}(1)}\right) \in L^{2}\left(S_{n}(\Lambda)\right)$ is an isometry. Consider the self-adjoint operator $H\left(S_{n}(\Lambda)\right)$ in $L^{2}\left(S_{n}(\Lambda)\right)$ given by the RHS of $(0.1)$ with the Dirichlet boundary conditions on $\partial S_{n}(\Lambda)$. It is not hard to check that $H\left(S_{n}(\Lambda)\right)=T_{n} H_{-, n}(\Lambda) T_{n}^{*}$. Hence,

$$
\operatorname{tr}_{L^{2}-\left(A^{n}\right)} \exp \left[-\beta H_{-, n}(\Lambda)\right]=\int_{S_{n}(\Lambda)} d \bar{x}^{n} \int d P_{\bar{x}^{n}, \bar{x}^{n}}^{\beta}\left(\bar{\omega}^{n}\right) \alpha_{S_{n}(\Lambda)}\left(\bar{\omega}^{n}\right) \exp \left[-U\left(\bar{\omega}^{n}\right)\right] .
$$

Now (3.1b) is obtained by the summation procedure and a simple change of notations.

We are now prepared to prove Proposition 1.1.

Proof of Proposition 1.1. Since $\alpha_{-}^{0} \leqq 1$, for the both cases $\varepsilon= \pm, \Xi_{\Lambda}^{\varepsilon}$ is less then or equal to the RHS of [7, (2.16)]. Since $\Phi \geqq 0$, the functional $U$ is also non-negative. Then $[7,(2.17)]$ (with $B=0$ ) gives :

$$
\Xi_{\Lambda} \leqq \exp \left[(2 \pi \beta)^{-1 / 2}|\Lambda| e^{\beta \mu}\right]
$$

which is finite for all $\beta \in R_{+}^{1}$ and $\mu \in R^{1}$. Q.E.D.

It is convenient to introduce the "fictitious" functional $\alpha_{+}^{0} \equiv 1$. Then we can write the unique formula for $\varepsilon= \pm$ :

$$
\Xi_{\Lambda}=\int_{[A]} d Y \alpha_{\Lambda}(Y) \alpha^{0}(Y) \exp [-U(Y)+\beta \mu n(Y)] .
$$

Let $\Omega \subset A$ be a b.B.s; the kernels $\Xi_{\Lambda}^{\Omega}\left(\bar{x}^{n}, \bar{y}^{n}\right), \bar{x}^{n}, \bar{y}^{n} \in \Omega^{n}, n=0,1, \ldots$, are defined by ${ }^{4}$

$$
\begin{aligned}
\Xi_{\Lambda}^{\Omega}\left(\bar{x}^{n}, \bar{y}^{n}\right)= & \varepsilon \varepsilon^{|\pi<|} \frac{e^{\beta \mu n}}{n !} \int_{[\Lambda \mid \Omega]]} d Y \int_{\left[\bar{x}^{n}, \bar{y}^{n}\right]_{<}} d X \alpha_{\Lambda}(X, Y) \\
& \cdot \alpha^{0}(X, Y) \exp [-U(X, Y)+\beta \mu n(Y)] .
\end{aligned}
$$

4 In what follows we shall use the notation $[\Theta]$ for denoting the space of the finite sets of simple closed loops $\omega$ starting in $\Theta$ for arbitrary b.B.s. $\Theta \subset R^{1}$ 
Here $\pi_{<}=\pi_{<}\left(\bar{x}^{n}, \bar{y}^{n}\right)$ is a permutation of $n^{\prime}$ th order satisfying the condition: $x_{i}$ $\leqq x_{j} \Rightarrow y_{\pi<i} \leqq y_{\pi_{<j}}, 1 \leqq i, j \leqq n, i \neq j$, and $\left[\bar{x}^{n}, \bar{y}^{n}\right]_{<}$is the collection of families $X$ $=\left(\omega_{1}, \ldots, \omega_{n}\right)$ of simple (time length $\left.\beta\right)$ trajectories with $\omega_{i}(0)=x_{i}, \omega_{i}(\beta)=y_{\pi<i}$, and finally, $d X=d P_{\tilde{x}^{n}, \pi<\bar{y}^{n}}^{\beta}(X)$.

Proposition 3.3. The following representation holds:

$$
\varrho_{\Lambda}^{\Omega}\left(\bar{x}^{n}, \bar{y}^{n}\right)=\left(\Xi_{\Lambda}\right)^{-1} \Xi_{\Lambda}^{\Omega}\left(\bar{x}^{n}, \bar{y}^{n}\right) .
$$

Proof. We omit the detailized proof: (3.2) and (3.3) are analogous to [7, (2.10)(2.15)]. The change of the domain of integration $f d Y([\Lambda \backslash \Omega]$ in (3.3) contrary to $[\Lambda]$ in [7]) is caused by the difference of the traces in the definitions of our $g_{A}^{\Omega}$ and of the RDM in [7]. The functional $\alpha^{0}$ in (3.2) plays the same role as in (3.1).

Notice that the kernel (3.3) is a continuous function of $\bar{x}^{n}, \bar{y}^{n}$. This follows easely from (3.1) and (3.2) in view of properties of the Wiener measure and the continuity of the function $\Phi$. Hence, by the Mercer theorem,

$$
\sum_{n=0}^{\infty} \int_{\Omega^{n}} d \bar{x}^{n} \varrho_{\Lambda}^{\Omega}\left(\bar{x}^{n}, \bar{x}^{n}\right)=\operatorname{tr}_{\mathscr{H}(\Omega)} g_{\Lambda}^{\Omega}=1
$$

It is useful to write down bounds for the kernels $Q_{A}^{\Omega}(\mathrm{cf}$. [7, Section 2.1]):

$$
\begin{aligned}
\left|\varrho_{\Lambda}^{\Omega}\left(\bar{x}^{n}, \bar{y}^{n}\right)\right| & \leqq \Psi\left(\bar{x}^{n}, \bar{y}^{n}\right)=\int_{\left[x^{n}, y^{n}\right]<} d X e^{\beta \mu n} \frac{1}{n !} \\
& \leqq \frac{1}{n !} c_{0}^{n}, \quad n=0,1, \ldots,
\end{aligned}
$$

where $c_{0} \in R_{+}^{1}$ depends on $\beta$ and $\mu$. Moreover,

$$
\sum_{n=0}^{\infty} \int_{\Omega^{n}} d \Omega^{n} \bar{x}^{n} d \bar{y}^{n} \Psi\left(\bar{x}^{n}, \bar{y}^{n}\right)^{2}<\infty, \quad \sum_{n=0}^{\infty} \int_{\Omega^{n}} d \bar{x}^{n} \Psi\left(\bar{x}^{n}, \bar{x}^{n}\right)<\infty .
$$

We conclude this Section by indicating two "Poissonian" properties of the measure $d Y$ repeatedly used below. The first of them is identity [7, (2.14)], and the second one is given by

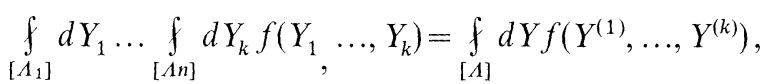

where $\Lambda_{1}, \ldots, \Lambda_{k}$ are b.B.s., $\bigcup_{s=1}^{k} \Lambda_{s}=\Lambda, \Lambda_{i} \cap \Lambda_{j} \neq \emptyset$ for $i \neq j$ and $Y^{(i)}$ is the part of $Y$ including those loops $\omega$ which start in $A_{i}, 1 \leqq i, j \leqq k$. The integral in the LHS of (3.6) will be denoted as $\int_{[\Lambda]} d\left(Y_{1}, \ldots, Y_{k}\right)$.

\section{Convergence of the Kernels $\varrho_{A}^{\Omega}$}

In this section we reduce Theorems 3 and 4 to statements about the kernels $\varrho_{\Lambda}^{\Omega}$. Theorem 5. For any b.B.s. $\Omega \subset R^{1}$ and all $\bar{x}^{n}, \bar{y}^{n} \in \Omega^{n}, n=0,1, \ldots$, there exist the limit

$$
\lim _{\Lambda \rightarrow R^{1}} \varrho_{\Lambda}^{\Omega}\left(\bar{x}^{n}, \bar{y}^{n}\right)=\varrho^{\Omega}\left(\bar{x}^{n}, \bar{y}^{n}\right) ;
$$

for fixed $\Omega$ and $n$ the convergence is uniform in $\bar{x}^{n}, \bar{y}^{n} \in \Omega^{n}$. 
The limit operator $g^{\Omega}$ in $\mathscr{H}(\Omega)$ is given by $g^{\Omega} f=\bigoplus_{n=0}^{\infty} \tilde{f}_{n}$, where $\tilde{f}_{n}$ consides with the RHS of (1.3) where $\varrho_{A}^{\Omega}$ is replaced by the limit kernel $\varrho^{\Omega}$.

Given $a \in R^{1}$, denote by $\bar{a}_{0}^{n}$ the vector $(a, \ldots, a) \in R^{n}$. Let $\Omega_{1}, \Omega_{2} \subset R^{1}$ be b.B.s. and $a$ be chosen so that $\Omega_{1} \cap\left(\Omega_{2}+a\right)=\emptyset$. Let $\varrho^{\Omega(a)}$ denote the kernel of the operator $\tilde{g}^{\Omega(a)}$ in $\mathscr{H}\left(\Omega_{1}\right) \otimes \mathscr{H}\left(\Omega_{2}\right)$. Clearly,

$$
\begin{aligned}
& \varrho^{\Omega(a)}\left(\bar{x}_{1}^{n_{1}}, \bar{x}_{2}^{n_{2}} ; \bar{y}_{1}^{n_{1}}, \bar{y}_{2}^{n_{2}}\right)=\varrho^{\Omega(a)}\left(\bar{x}_{1}^{n_{1}}, \bar{x}_{2}^{n_{2}}+\bar{a}_{0}^{n_{2}} ; \bar{y}_{1}^{n_{1}}, \bar{y}_{2}^{n_{2}}+\bar{a}_{0}^{n_{2}}\right), \\
& \bar{x}_{i}^{n_{2}}, \bar{y}_{i}^{n_{2}} \in \Omega_{i}^{n_{2}}, n_{i}=0,1, \ldots, i=1,2 .
\end{aligned}
$$

Theorem 6. i) For any b.B.s. $\Omega \subset R^{1}$ and $a \in R^{1}$ and for all $\bar{x}^{n}, \bar{y}^{n} \in \Omega^{n}, n=0,1, \ldots$

$\varrho^{\Omega}\left(\bar{x}^{n}, \bar{y}^{n}\right)=\varrho^{\Omega+a}\left(\bar{x}^{n}+\bar{a}_{0}^{n}, \bar{y}^{n}+\bar{a}_{0}^{n}\right)$.

ii) For any b.B.s. $\Omega_{1}, \Omega_{2} \subset R^{1}$ and all $\bar{x}_{i}^{n_{l}}, \bar{y}_{i}^{n_{l}} \in \Omega_{i}^{n_{t}}, n_{i}=0,1, \ldots, i=1,2$,

$$
\lim _{a \rightarrow \infty} \varrho^{\Omega(a)}\left(\bar{x}_{1}^{n_{1}}, \bar{x}_{2}^{n_{2}} ; \bar{y}_{1}^{n_{1}}, \bar{y}_{2}^{n_{2}}\right)=\varrho^{\Omega_{1}}\left(\bar{x}_{1}^{n_{1}}, \bar{y}_{1}^{n_{1}}\right) \varrho^{\Omega_{2}}\left(\bar{x}_{2}^{n_{2}}, \bar{y}_{2}^{n_{2}}\right) \text {. }
$$

Theorem 3 follows from Theorem 5. In fact, the limit kernels $\varrho^{\Omega}\left(\bar{x}^{n}, \bar{y}^{n}\right)$ obey (3.4) and, due to (3.5), the limit operators $g^{\Omega}$ are of the Hilbert-Schmidt class. Moreover,

$$
\left[\varrho^{\Omega}\left(\bar{x}^{n}, \bar{y}^{n}\right)-\varrho_{\Lambda}^{\Omega}\left(\bar{x}^{n}, \bar{y}^{n}\right)\right]^{2} \leqq 4 \Psi\left(\bar{x}^{n}, \bar{y}^{n}\right)^{2}
$$

and hence, by Theorem 5 and the Lebesque convergence theorem, (2.1) holds. Clearly, $g^{\Omega}$ is non-negative. Since the convergence in (4.1) is uniform, the limit kernel is continuous. By the Mercer theorem, $\operatorname{tr}_{\mathscr{H}(\Omega)} g^{\Omega}=\sum_{n=0}^{\infty} \int_{\Omega^{n}} d \bar{x}^{n} Q^{\Omega}\left(\bar{x}^{n}, \bar{x}^{n}\right)$, and hence, by the Lebesgue convergence theorem, $\operatorname{tr}_{\mathscr{H}(\Omega)} g^{\Omega}=1$.

Similarly, Theorem 4 is a consequence of Theorem 6 . The relation between the statements i) of the both theorems is obvious, and now we explain how to get ii) of Theorem 4 having ii) of Theorem 6 . The kernel $\varrho^{\Omega(a)}$ satisfies a bound analogous to (3.4); its RHS is denoted as $\Psi_{a}\left(\bar{x}_{1}^{n_{1}}, \bar{x}_{2}^{n_{2}} ; \bar{y}_{1}^{n_{1}}, \bar{y}_{2}^{n_{2}}\right)$. It is not hard to check that, for fixed $\Omega_{i}, \bar{x}_{i}^{n_{2}}$ and $\bar{y}_{i}^{n_{i}}, i=1,2, \Psi_{a}$ monotonically decreases w.r.t. a for $a \geqq a^{(0)}\left(a^{(0)}\right.$ depends on $\Omega_{1}, \Omega_{2}$ ). Hence, for such $a$

$$
\begin{aligned}
& {\left[\varrho^{\Omega(a)}\left(\bar{a}_{1}^{n_{1}}, \bar{x}_{2}^{n_{2}} ; \bar{y}_{1}^{n_{1}}, \bar{y}_{2}^{n_{2}}\right)-\varrho^{\Omega_{1}}\left(\bar{x}_{1}^{n_{1}}, \bar{y}_{1}^{n_{1}}\right) \varrho^{\Omega_{2}}\left(\bar{x}_{2}^{n_{2}}, \bar{y}_{2}^{n_{2}}\right)\right]^{2}} \\
& \quad \leqq 2\left[\Psi_{a^{(0)}}\left(\bar{x}_{1}^{n_{1}}, \bar{x}_{2}^{n_{2}} ; \bar{y}_{1}^{n_{1}}, \bar{y}_{2}^{n_{2}}\right)^{2}+\Psi\left(\bar{x}_{1}^{n_{1}}, \bar{y}_{1}^{n_{1}}\right) \Psi\left(\bar{x}_{2}^{n_{2}}, \bar{y}_{2}^{n_{2}}\right)\right] .
\end{aligned}
$$

The kernel $\Psi_{a^{(0)}}$ satisfies bound (3.5) as well as $\Psi$. Due to the Lebesgue convergence theorem, (4.2) and (4.3) imply (2.4).

In Section 5-8 we give the proof of Theorem 5 .

\section{Preliminary Estimates}

To be definite we suppose below that $n \geqq 1$ in $(4.1)$; the reader can repeat all arguments for $n=0$ without difficulties. We also can suppose without loss of generality that $\bar{x}^{n}, \bar{y}^{n}$ in (4.1) are chosen so that $\pi_{<}$is the identity permutation and omit the index $<$ in $\left[\bar{x}^{n}, \bar{y}^{n}\right]_{<}$. Our arguments will be applied to the both cases $\varepsilon=$ 
\pm simultaneously. Fix a b.B.s. $\Omega \subset R^{1}$ and denote $\gamma_{-}=\inf _{x \in \Omega} x, \gamma_{+}=\sup _{x \in \Omega} x, \gamma=1 / 2(\gamma$ $\left.+\gamma_{+}\right)$. Next fix $t_{1}, t_{2} \in R_{+}^{1}$. Let $\Lambda=\left(a_{-}, a_{+}\right)$be a bounded interval with

$$
a_{-} \leqq \gamma-\left(t_{1}+t_{2}\right), a_{+} \geqq \gamma+\left(t_{1}+t_{2}\right) ;
$$

consider the partition of $\Lambda$ into four parts ${ }^{5}$ :

$$
\begin{aligned}
& \Lambda_{I}(-)=\left[\gamma-t_{2}, \gamma\right], \Lambda_{I}(+)=\left[\gamma, \gamma+t_{2}\right], \\
& \Lambda_{E}(-)=\left(a_{-}, \gamma-t_{2}\right), \Lambda_{E}(+)=\left(\gamma+t_{2}, a_{+}\right) .
\end{aligned}
$$

For brevity, denote: $\Lambda_{\#}=\Lambda_{\text {\#\# }}(-) \cup \Lambda_{\text {\#\# }}(+)$, \# $=I, E$. Elements of $\left[\Lambda_{\#}\right]$ i.e., finite sets of simple closed loops starting in $\Lambda_{\#}$ are denoted by $Y_{\#}$.

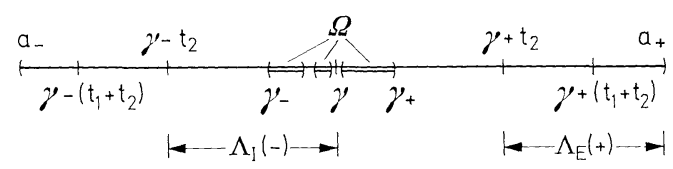

Fig. 1. The partition

Fix $\bar{x}^{n}, \bar{y}^{n} \in \Omega^{n}$ and let

$$
\begin{aligned}
& \Xi_{\Lambda, \Lambda_{E}}^{(1)}=\int_{[\Lambda]} d\left(Y_{E}, Y_{I}\right) \alpha_{\Lambda}\left(Y_{E}\right) \alpha^{0}\left(Y_{I}, Y_{E}\right) \\
& \cdot \exp \left[-U\left(Y_{I}, Y_{E}\right)+\beta \mu n\left(Y_{I}\right)+\beta \mu n\left(Y_{E}\right)\right], \\
& \Xi_{\Lambda, \Lambda_{E}}^{(1), \Omega}\left(\bar{x}^{n}, \bar{y}^{n}\right)=\frac{e^{\beta \mu n}}{n !} \int_{[\Lambda \backslash \Omega]} d\left(Y_{E}, Y_{I}\right) \int_{\left[x^{n}, y^{n}\right]} d X \alpha_{\Lambda}\left(Y_{E}\right) \\
& \cdot \alpha^{0}\left(X, Y_{I}, Y_{E}\right) \exp \left[-U\left(X, Y_{I}, Y_{E}\right)+\beta \mu n\left(Y_{I}\right)+\beta \mu n\left(Y_{E}\right)\right],
\end{aligned}
$$

and

$$
\varrho_{\Lambda, \lambda_{E}}^{(1), \Omega}\left(\bar{x}^{n}, \bar{y}^{n}\right)=\left(\Xi_{\Lambda, \lambda_{E}}^{(1)}\right)^{-1} \Xi_{\Lambda, \Lambda_{E}}^{(1), \Omega}\left(\bar{x}^{n}, \bar{y}^{n}\right) .
$$

The RHS of (5.3) and (5.4) differ from those of (3.1) and (3.2) in view of the absence of $\alpha_{A}\left(Y_{I}\right)$ and $\alpha_{A}(X)$.

Lemma 5.1. There exist constants $q_{1}, c_{1}, c_{2} \in R_{+}^{1}$ depending only on $\beta$ and $\mu$ such that ${ }^{6}$

$$
\left|\varrho_{A, A_{E}}^{(1) \Omega}\left(\bar{x}^{n}, \bar{y}^{n}\right)-\varrho_{A}^{\Omega}\left(\bar{x}^{n}, \bar{y}^{n}\right)\right| \leqq n c_{0}^{n} c_{1} \exp \left(-c_{2} t_{1}^{2}\right)
$$

whenever $t_{1}>q_{1}$.

Proof of Lemma 5.1. Our proof is similar to that of Lemma 2.1 in [14]. Clearly, $\left(\Xi_{\Lambda, A_{E}}^{(1)}\right)^{-1} \Xi_{\Lambda} \leqq 1$. We claim that $\left(\Xi_{\Lambda, A_{E}}^{(1)}\right)^{-1} \Xi_{\Lambda} \geqq 1-c_{1}^{\prime} \exp \left(-c_{2} t_{1}^{2}\right)$ where $c_{1}^{\prime}, c_{2} \in R_{+}^{1}$ depend only on $\beta$ and $\mu$. In fact, replace $\left[1-\alpha_{A}\left(Y_{I}\right)\right]$ by the sum $\sum_{\dot{\omega} \in Y_{I}}\left[1-\alpha_{A}(\omega)\right]$ in

5 The indices have the following meaning: $I=$ "internal", $E=$ "external"

6 The factor $1 / n$ ! is omitted for brevity from the bounds we establish below 
the integrand for $\Xi_{\Lambda, \Lambda_{E}}^{(1)}-\Xi_{\Lambda}$. Using $[7,(2.14)]$ we then have

$$
\begin{gathered}
\Xi_{\Lambda, \Lambda_{E}}^{(1)}-\Xi_{\Lambda} \leqq e^{\beta \mu} \int_{\left[\Lambda_{I}\right]_{1}} d \omega\left[1-\alpha_{\Lambda}(\omega)\right] \int_{[\Lambda]} d\left(Y_{E}, Y_{I}\right) \alpha_{\Lambda}\left(Y_{E}\right) \\
\cdot \exp \left[-U\left(\omega, Y_{I}, Y_{E}\right)+\beta \mu n\left(Y_{I}\right)+\beta \mu n\left(Y_{E}\right)\right]
\end{gathered}
$$

where $\left[\Lambda_{I}\right]_{1}$ is the set of simple closed loops $\omega$ with $\omega(0) \in \Lambda_{I}, d \omega$ is given by $[7$, (2.32)]. Since $\Phi \geqq 0, U\left(\omega, Y_{I}, Y_{E}\right) \geqq U\left(Y_{I}, Y_{E}\right)$, and the integral $\int d\left(Y_{E}, Y_{I}\right)$ is less than or equal to $\Xi_{\Lambda, A_{E}}^{(1)}$. The integral $\int d \omega$ is estimated using the standard formula for the probability of deviation of a Wiener trajectory (see, e.g., [13, Chapter VI, $\S 5$, Corollary 2]) and does not exceed $c_{1}^{\prime} \exp \left(-c_{2} t_{1}^{2}\right)$. This gives the estimate claimed above.

Take $q_{1}$ so large that $c_{1}^{\prime} \exp \left(-c_{2} t_{1}^{2}\right) \leqq 1 / 2$. Then the inverse ratio $\left(\Xi_{A}\right)^{-1} \Xi_{\Lambda, A_{E}}^{(1)}$ $\leqq 1+2 c_{1}^{\prime} \exp \left(-c_{2} t_{1}^{2}\right)$ and the LHS of $(5.6)$ is

$$
\begin{aligned}
\leqq & {\left[\varrho_{\Lambda, \Lambda_{E}}^{(1)}\left(\bar{x}^{n}, \bar{y}^{n}\right)-\left(\Xi_{\Lambda, \Lambda_{E}}^{(1)}\right)^{-1} \Xi_{\Lambda}^{\Omega}\left(\bar{x}^{n}, \bar{y}^{n}\right)\right] } \\
& +\left(\Xi_{\Lambda, \Lambda_{E}}^{(1)}\right)^{-1} \Xi_{\Lambda}^{\Omega}\left(\bar{x}^{n}, \bar{y}^{n}\right)\left[\left(\Xi_{\Lambda}\right)^{-1} \Xi_{\Lambda, \Lambda_{E}}^{(1)}-1\right],
\end{aligned}
$$

where the second term is $\leqq 2 c_{0}^{n} c_{1}^{\prime} \exp \left(-c_{2} t_{1}^{2}\right)$. It remains to estimate the first term in (5.7), i.e., the difference $\bar{\Xi}_{\Lambda, A_{E}}^{(1), \Omega}\left(\bar{x}^{n}, \bar{y}^{n}\right)-\Xi_{A}^{\Omega}\left(\bar{x}^{n}, \bar{y}^{n}\right)$. The arguments similar to those used above give that it is less than or equals $n c_{0}^{n} c_{1}^{\prime} \exp \left(-c_{2} t_{1}^{2}\right)$ (the factor $n$ appears in view of the bound $\left.1-\alpha_{\Lambda}\left(X, Y_{I}\right) \leqq \sum_{\omega \in X}\left[1-\alpha_{\Lambda}(\tilde{\omega})\right]+\sum_{\omega \in Y_{I}}\left[1-\alpha_{\Lambda}(\omega)\right]\right)$. This gives
the assertion of Lemma 5.1 .

\section{Local Particle Number Bounds}

For technical reasons we now assume that $\gamma, \gamma_{ \pm}$, and $a_{ \pm}$are integers. The arguments below may be easily extended to the general case. For the rest of the paper we also choose $t_{2}$ to be of the form $\bar{\gamma}+\bar{t}\left(\operatorname{IP}\left(c_{3} \ln \bar{t}\right)+3\right)$ where $\gamma=1 / 2\left(\gamma_{+}\right.$ $\left.-\gamma_{-}\right), \bar{t} \geqq 1$ is an integer, IP denotes the integer part, and $c_{3}=c_{3}(\beta, \mu)$ will be indicated in Section 8.

Every set (5.2) is divided into the unit intervals $(j, j+1)$ where $j$ is an integer. We denote them as $\Delta_{\#}( \pm ; k), k=1,2, \ldots, \#=I, E$. The order of the numeration of the unit intervals is different for different sets (5.2), and we will choose it in Section 7.

Given $Y \in[\Lambda]$, denote by $l(Y)$ the maximal deviation $\max _{\omega \in Y} \sup _{0 \leqq t \leqq \beta}|\tilde{\omega}(t)-\tilde{\omega}(0)|$. Given $Y_{\#} \in\left[\Lambda_{\#}\right]$, denote by $Y_{\#}( \pm, k)$ its "local" part consisting of loops $\omega \in Y_{\text {\# }}$ starting in $\Delta_{\#}( \pm, k)$ :

$$
Y_{\#}( \pm, k)=Y_{\#} \cap\left[\Delta_{\#}( \pm, k)\right], k=1,2, \ldots, \#=I, E .
$$

Next fix two sequences of positive integers $\boldsymbol{N}=\left(n_{0}, n_{1}, \ldots\right)$ and $\boldsymbol{L}=\left(l_{0}, l_{1}, \ldots\right)$ and set: $n_{k}^{(I)}=n_{0}, l_{k}^{(I)}=l_{0}$ and $n_{k}^{(E)}=n_{k}, l_{k}^{(E)}=l_{k}, k=1,2, \ldots$. We say that $Y_{\#}$ is $(\boldsymbol{N}, \boldsymbol{L})$ regular if $n\left(Y_{\#}( \pm ; k)\right) \leqq n_{k}^{(\#)}, l\left(Y_{\#}( \pm ; k)\right) \leqq l_{k}^{(\#)}, k=1,2, \ldots, \quad \#=I, E$. Similarly, $X \in\left[\bar{x}^{n}, \bar{y}^{n}\right]$ is called $(\boldsymbol{N}, \boldsymbol{L})$-regular if $n<n_{0}$ and $l(X)=\max _{\tilde{\omega} \in X} \sup _{t_{1}, t_{2}}\left|\tilde{\omega}\left(t_{1}\right)-\tilde{\omega}\left(t_{2}\right)\right|<l_{0}$. Let the indicator functional $\chi_{N, L}$ be given by

$$
\chi_{\boldsymbol{N}, \boldsymbol{L}}\left(X, Y_{I}, Y_{E}\right)=\left\{\begin{array}{l}
1, \text { if } X \text { and } Y_{\#} \text { are }(\boldsymbol{N}, \boldsymbol{L}) \text {-regular, \#=I,E } \\
0, \text { otherwise. }
\end{array}\right.
$$


Denote

$$
\begin{aligned}
& \Xi_{\Lambda, \boldsymbol{N}, \boldsymbol{L}}^{(2)}=\int_{[\Lambda]} d\left(Y_{E}, Y_{I}\right) \chi_{\boldsymbol{N}, \boldsymbol{L}}\left(Y_{I}, Y_{E}\right) \alpha_{\Lambda}\left(Y_{E}\right) \alpha^{0}\left(Y_{I}, Y_{E}\right) \\
& \cdot \exp \left[-U\left(Y_{I}, Y_{E}\right)+\beta \mu n\left(Y_{I}\right)+\beta \mu n\left(Y_{E}\right)\right], \\
& \Xi_{\Lambda, \boldsymbol{N}, \boldsymbol{L}}^{(2), \Omega}\left(\bar{x}^{n}, \bar{y}^{n}\right)=\frac{e^{\beta \mu n}}{n !} \int_{[\Lambda \backslash \Omega]} d\left(Y_{E}, Y_{I}\right) \int_{\left[\bar{x}^{n}, \bar{y}^{n}\right]} d X \chi_{\boldsymbol{N}, \boldsymbol{L}}\left(X, Y_{I}, Y_{E}\right) \alpha_{\Lambda}\left(Y_{E}\right) \\
& \cdot \alpha^{0}\left(X, Y_{I}, Y_{E}\right) \exp \left[-U\left(X, Y_{I}, Y_{E}\right)+\beta \mu n\left(Y_{I}\right)+\beta \mu n\left(Y_{E}\right)\right]
\end{aligned}
$$

and

$$
\varrho_{\Lambda, \boldsymbol{N}, \boldsymbol{L}}^{(2) \Omega}\left(\bar{x}^{n}, \bar{y}^{n}\right)=\left(\Xi_{\Lambda, \boldsymbol{N}, \boldsymbol{L}}^{(2)}\right)^{-1} \Xi_{\Lambda, \boldsymbol{N}, \boldsymbol{L}}^{(2), \Omega}\left(\bar{x}^{n}, \bar{y}^{n}\right) .
$$

We consider a particular case by choosing $n_{0}=l_{0}=\operatorname{IP}\left(1 / 6 c_{3} \ln \bar{t}\right)$ and $n_{k}=n_{0}$ $+k, l_{k}=l_{0}+1 / 2 k, k=1,2, \ldots$ The LHS's of (6.1)-(6.3) are then denoted as $\Xi_{\Lambda, \bar{t}}^{(2)}$, $\Xi_{\Lambda, \bar{t}}^{(2), \Omega}\left(\bar{x}^{n}, \bar{y}^{n}\right)$ and $\varrho_{\Lambda, \bar{t}}^{(2), \Omega}\left(\bar{x}^{n}, \bar{y}^{n}\right)$ respectively.

Lemma 6.1. There exist constants $q_{2}, c_{4}, c_{5} \in R_{+}^{1}$ depending only on $\beta$ and $\mu$ such that

$$
\begin{aligned}
& \left|\varrho_{\Lambda, \bar{t}}^{(2), \Omega}\left(\bar{x}^{n}, \bar{y}^{n}\right)-\varrho_{\Lambda, A_{E}}^{(1), \Omega}\left(\bar{x}^{n}, \bar{y}^{n}\right)\right| \\
& \quad \leqq n c_{0}^{n} c_{4}(\bar{t} \ln \bar{t}) \exp \left(-c_{5}(\ln \bar{t}) \ln \ln \bar{t}\right)
\end{aligned}
$$

whenever $\bar{t} \geqq q_{2}$ and $n_{0}>n$.

Proof. First, assume we have the general sequences $\boldsymbol{N}, \boldsymbol{L}$. Clearly, $\left(\Xi_{\Lambda, \Lambda_{E}}^{(1)}\right)^{-1} \Xi_{\Lambda, \boldsymbol{N}, \boldsymbol{L}}^{(2)}$ $\leqq 1$. We claim that

$$
\begin{aligned}
& \left(\Xi_{\Lambda, \Lambda_{E}}^{(1)}\right)^{-1} \Xi_{\Lambda, \boldsymbol{N}, \boldsymbol{L}}^{(2)} \geqq 1-c_{4}^{\prime \prime}\left[t_{2}\left(\exp \left(-c_{2} l_{0}^{2}\right)+\exp \left(-c_{5}^{\prime} n_{0} \ln n_{0}\right)\right)\right] \\
& \left.\quad+\sum_{k=1}^{\infty}\left(\exp \left(-c_{2} l_{k}^{2}\right)+\exp \left(-c_{5}^{\prime} n_{k} \ln n_{k}\right)\right)\right],
\end{aligned}
$$

where $c_{4}^{\prime \prime}, c_{5}^{\prime} \in R_{+}^{1}$. In fact, the factor $\left[1-\chi_{N, \boldsymbol{L}}\left(Y_{I}, Y_{E}\right)\right]$ in the integrand for $\Xi_{\Lambda, A_{E}}^{(1)}$ $-\Xi_{\Lambda, \boldsymbol{N}, \boldsymbol{L}}^{(2)}$ does not exceed $\sum\left[1-\chi_{\boldsymbol{N}, \boldsymbol{L}}\left(Y_{\#}(x ; k)\right)\right]$ where the sum is taken over all \# $=E, I, x= \pm$ and $k=1,2, \ldots$. Consider separately a single term corresponding to a given $\Delta_{\#}(\varkappa ; k)$. To estimate the single term we write the integral $f_{[\Lambda]} d\left(Y_{E}, Y_{I}\right)$ as $\int_{[\Lambda \backslash \Delta \#(\varkappa ; k)]} d\left(Y_{E}, Y_{I}\right) \int_{[\Delta \#(\varkappa ; k)]} d Y^{0}$ and replace $U\left(Y^{0}, Y_{I}, Y_{E}\right)$ by $U\left(Y_{I}, Y_{E}\right)$ and the indicators $\alpha\left(Y^{0}, \cdot\right)$ by $\alpha(\cdot)$. Since $U\left(Y^{0}, Y_{I}, Y_{E}\right) \geqq U\left(Y_{I}, Y_{E}\right)$, this leads at an expression which is more than or equal to the starting one. Now the integral $f d Y^{0}$ may be separated, and our single term is bounded by

$$
\int_{[\Delta \#(\varkappa ; k)]} d Y^{0}\left[1-\chi_{\boldsymbol{N}, \boldsymbol{L}}\left(Y^{0}\right)\right] \exp \left[\beta \mu n\left(Y^{0}\right)\right] \Xi_{\Lambda, \Lambda_{E}}^{(1)} .
$$

The factor $\left[1-\chi_{\boldsymbol{N}, \boldsymbol{L}}\left(Y^{0}\right)\right]$ does not vanish iff either $n\left(Y^{0}\right)>n_{k}^{(\#)}$ or $l\left(Y^{0}\right)>l_{k}^{(\#)}$. The first possibility gives a contribution which is estimated by $\exp \left(-c_{5}^{\prime} n_{k}^{(\#)} \ln n_{k}^{(\#)}\right)$ (this may be easily derived from the definition of the measure $d Y$ ). The contribution of the second possibility is estimated using the same arguments as in the preceding section and does not exceed $c_{4}^{\prime \prime \prime} \exp \left(-c_{2}\left(l_{k}^{(\#)}\right)^{2}\right)$. Taking $n_{k}^{(\#)}$ and $l_{k}^{(\#)}$ as it was indicated above and suming the bounds obtained over \#, $x$ and $k$, we arrive at (6.5). 
With our choice of $t_{2}, n_{k}$, and $l_{k}$, bound (6.5) takes the form

$$
\left(\Xi_{\Lambda, A_{E}}^{(1)}\right)^{-1} \Xi_{\Lambda}^{(2)} \geqq 1-c_{4}^{\prime}(\bar{t} \ln \bar{t}) \exp \left(-c_{5}(\ln \bar{t}) \ln \ln \bar{t}\right) \equiv 1-\eta(\bar{t})
$$

(we omit intermediate calculations). Take $q_{2}$ so large that $\eta(t) \leqq 1 / 2$ for all $t>q_{2}$. Then for $\bar{t} \geqq q_{2}$

$$
O \leqq\left(\Xi_{\Lambda, \bar{t}}^{(2)}\right)^{-1} \Xi_{\Lambda, \Lambda_{E}}^{(1)}-1 \leqq 2 \eta(\bar{t}) .
$$

Now the LHS of (6.4) is

$$
\begin{aligned}
& \leqq\left[\varrho_{\Lambda, \bar{t}}^{(2), \Omega}\left(\bar{x}^{n}, \bar{y}^{n}\right)-\left(\Xi_{\Lambda, \Lambda_{E}}^{(1)}\right)^{-1} \Xi_{\Lambda, t}^{(2), \Omega}\left(\bar{x}^{n}, \bar{y}^{n}\right)\right] \\
& +\left(\Xi_{\Lambda, \Lambda_{E}}^{(1)}\right)^{-1} \Xi_{\Lambda, \bar{t}}^{(2), \Omega}\left(\bar{x}^{n}, \bar{y}^{n}\right)\left[\left(\Xi_{\Lambda, \bar{t}}^{(2)}\right)^{-1} \Xi_{\Lambda, \Lambda_{E}}^{(1)}-1\right] .
\end{aligned}
$$

The second term of $(6.7)$ is less than or equal to $2 c_{0}^{n} \eta(\bar{t})$. To estimate the first term we use arguments similar to those we used above (cf. the corresponding part of the proof of Lemma 5.1). The first term is bounded by $n c_{0}^{n} \eta(\bar{t})$. Lemma 6.1. is proved.

\section{A Cutoff Interaction}

Let us now choose the numeration of $\Delta_{\#}( \pm ; k)$ 's. Let $\delta_{+,+}=\delta_{-,-}=1, \delta_{+,-}=\delta_{-,+}$ $=0$. We set:

$$
\Delta_{E}( \pm ; k)=\left(\gamma \pm t_{2} \pm k-\delta_{ \pm,+}, \gamma \pm t_{2} \pm k+\delta_{ \pm,-}\right), \quad k=1,2, \ldots
$$

The order of $\Delta_{I}( \pm ; k)$ may be arbitrary (see Fig. 2).

The functional $U$ figuring in (6.1) and (6.2) and describing the "interaction" of trajectories is the sum $\sum u\left(\omega, \omega^{\prime}\right)$ of terms $u\left(\omega, \omega^{\prime}\right)=\int_{0}^{\beta} \Phi\left(\left|\omega(t)-\omega^{\prime}(t)\right|\right) d t$ giving the contritution of every pair of simple trajectories (see [7, Section 2.1]. We intriduce a new "cutoff" term $u^{(h)}\left(\omega, \omega^{\prime}\right)$ by

$$
u^{(h)}\left(\omega, \omega^{\prime}\right)=\left\{\begin{array}{l}
u\left(\omega, \omega^{\prime}\right) \text { if }\left|\omega(0)-\omega^{\prime}(0)\right|<h, \\
0, \text { otherwise. }
\end{array}\right.
$$

Next, define $U_{h}\left(Y_{I}, Y_{E}\right)\left[\right.$ resp., $\left.U_{h}\left(X, Y_{I}, Y_{E}\right)\right]$ as $U\left(Y_{I}, Y_{E}\right)\left[\right.$ resp., $\left.U\left(X, Y_{I}, Y_{E}\right)\right]$ with replacing the terms $u\left(\omega, \omega^{\prime}\right)$ by $u^{(h)}\left(\omega, \omega^{\prime}\right)$ in the following cases:

a) either $\omega$ or $\omega^{\prime}$ is a simple closed loop from $Y_{I}$,

b) $\omega$ belongs to $Y_{E}$ and $\omega^{\prime}$ belongs to $X$.

Let $\Xi_{\Lambda, \bar{t}}^{(3)}, \Xi_{\Lambda, \bar{t}}^{(3),}\left(\bar{x}^{n}, \bar{y}^{n}\right)$ and $\varrho_{\Lambda, \bar{t}}^{(3)}\left(\bar{x}^{n}, \bar{y}^{n}\right)$ be defined by formulas (6.1)-(6.3) with replacing the functionals $U$ by $U_{h}$ with $h=\operatorname{IP}\left(c_{3} \ln \bar{t}\right)+3$.

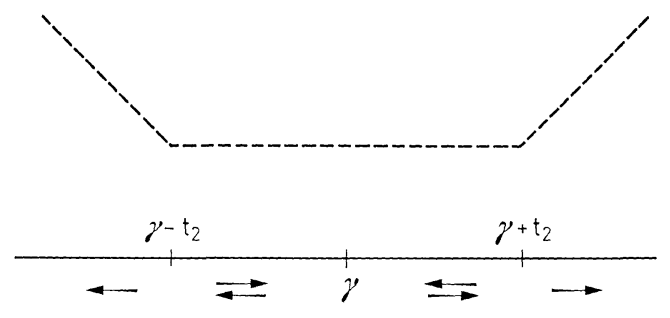

Fig. 2. Ordering and the graphs for $l_{k}^{(\#), n_{k}^{(\#)}}$ 
Lemma 7.1. The following bound holds:

$$
\left|\varrho_{\Lambda, \bar{t}}^{(3), \Omega}\left(\bar{x}^{n}, \bar{y}^{n}\right)-\varrho_{\Lambda . \bar{t}}^{(2), \Omega}\left(\bar{x}^{n}, \bar{y}^{n}\right)\right|<\xi(\bar{t}),
$$

where $\lim _{t \rightarrow \infty} \xi(t)=0$.

Proof. Clearly, $U_{h}\left(Y_{I}, Y_{E}\right) \leqq U\left(Y_{I}, Y_{E}\right)$. We wish to write down an appropriate upper bound for $U\left(Y_{I}, Y_{E}\right)-U_{h}\left(Y_{I}, Y_{E}\right)$ whenever $\chi_{\boldsymbol{N}, \boldsymbol{L}}\left(Y_{I}, Y_{E}\right)=1$.

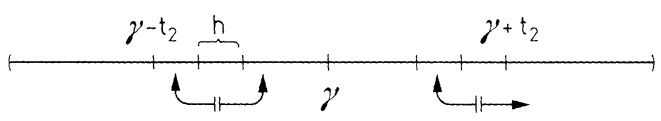

Fig. 3. The cutoffs. Broken narrows indicate the broken interaction

It follows from the definition of $U_{h}$ that $U\left(Y_{I}, Y_{E}\right)-U_{h}\left(Y_{I}, Y_{E}\right)$ is equal to

$$
\begin{aligned}
& 1 / 2 \sum_{\varkappa_{1}, \varkappa_{2}= \pm k_{1}, k_{2}:\left(\varkappa_{1} ; k_{1}\right) \neq\left(\varkappa_{2}, k_{2}\right)} \sum_{\omega_{1} \in Y_{I}\left(\varkappa_{1}, k_{1}\right), i=1,2}\left[u\left(\omega_{1}, \omega_{2}\right)-u^{(h)}\left(\omega^{1}, \omega_{2}\right)\right] \\
& +\sum_{x_{1}, \varkappa_{2}= \pm} \sum_{k_{1}, k_{2}} \sum_{\omega_{1} \in Y_{E}\left(\varkappa_{1} ; k_{1}\right), \omega_{2} \in Y_{I}\left(\varkappa_{2} ; k_{2}\right)}\left[u\left(\omega_{1}, \omega_{2}\right)-u^{(h)}\left(\omega_{1}, \omega_{2}\right)\right] .
\end{aligned}
$$

Suppose $\chi_{\boldsymbol{N}, \boldsymbol{L}}\left(Y_{I}, Y_{E}\right)=1$. First, consider the first line of (7.2). Every internal sum $\sum_{\omega_{1}, \omega_{2}}$ vanishes whenever $\hat{d}=\sup _{x_{1} \in \Delta_{I}\left(x_{t}, k_{\imath}\right)}\left|x_{1}-x_{2}\right| \leqq h$; otherwise, it does not exceed $\beta n_{0}^{2} \psi\left(\hat{d}_{1}\right)$, where $\hat{d}_{1}=\hat{d}-2-2 l_{0}$. With our choice of $n_{0}, l_{0}$ and $h$, we have: $\beta n_{0}^{2}$ $<c_{6}^{\prime \prime \prime}(\ln \bar{t})^{2}$, and the condition $\hat{d}^{\prime} h$ implies that $\hat{d}_{1}>2 / 3 c_{3} \ln \bar{t}$. Hence, the whole sum in the first line of (7.2) is bounded by $c_{6}^{\prime \prime} \bar{t}\left(\ln \bar{t}^{3} \sum_{k \geqq 2 / 3 c_{3} \ln \bar{t}} \psi(k)\right.$ where $c_{6}^{\prime \prime}$
$=c_{6}^{\prime \prime}(\beta, \mu) \in R_{+}^{1}$.

Similarly, every internal sum $\sum_{\omega_{1}, \omega_{2}}$ in the second line of (7.2) vanishes whenever $\hat{d} \leqq h$, otherwise, it is less than or equal to $\beta n_{0} n_{k_{1}} \psi\left(\hat{d}_{2}\right)$ where $\hat{d}_{2}=\hat{d}-2-l_{0}-l_{k_{1}}$. A simple analysis shows that with our choice of $n_{k}, l_{k}, h$ and the ordering of $\Delta( \pm, k)$ 's, we have: $\beta n_{0} n_{k} \leqq c_{6}^{\prime} t(\operatorname{In} \bar{t})(\operatorname{In} \bar{t}+k)$, and the condition $\hat{d}>h$ implies that $\hat{d}_{2}$ $>1 / 6 c_{3} \ln \bar{t}$. The whole sum in the second line of (7.2) is bounded by $c_{6}^{\prime}(\ln \bar{t})^{2} \sum_{k>1 / 6 c_{3} \ln \bar{t}} k^{2} \psi(k)$, where $c_{6}^{\prime}=c_{6}^{\prime}(\beta, \mu) \in R_{+}^{1}$. Finally, we obtain that

$$
U\left(Y_{I}, Y_{E}\right)-U_{h}\left(Y_{I}, Y_{E}\right) \leqq c_{6} \bar{t} \ln \bar{t} \sum_{k>1 / 6 c_{3} \ln \bar{t}} k^{2} \psi(k) \equiv \xi^{\prime}(\bar{t})
$$

where $\lim _{t \rightarrow \infty} \xi^{\prime}(t)=0$ due to the condition of decreasing $\psi(k)$.

Thus the bounds

$$
1 \leqq \frac{\exp \left[-U_{h}\left(Y_{I}, Y_{E}\right)\right]}{\exp \left[-U\left(Y_{I}, Y_{E}\right)\right]} \leqq \exp \left[\xi^{\prime}(\bar{t})\right]
$$

hold whenever $\chi_{N, \boldsymbol{L}}\left(Y_{I}, Y_{E}\right)=1$. 
Similar arguments allow us to establish similar bounds for $U_{h}\left(X, Y_{I}, Y_{E}\right)$ and $U\left(X, Y_{I}, Y_{E}\right)$ which hold whenever $\chi_{N, L}\left(X, Y_{I}, Y_{E}\right)=1$. This leads at the bounds

$$
1 \leqq \frac{\Xi_{A, \bar{t}}^{(2)}}{\Xi_{\Lambda, \bar{t}}^{(3)}}, \quad \frac{\Xi_{\Lambda, \bar{t}}^{(3) \Omega}\left(\bar{x}^{n}, \bar{y}^{n}\right)}{\Xi_{\Lambda, \bar{t}}^{(2), \Omega}\left(\bar{x}^{n}, \bar{y}^{n}\right)} \leqq \exp \left[\xi^{\prime}(t)\right] .
$$

Hence,

$$
\begin{aligned}
& \left|\varrho_{\Lambda, \bar{t}}^{(3), \Omega}\left(\bar{x}^{n}, \bar{y}^{n}\right)-\varrho_{\Lambda, \bar{t}}^{(2), \Omega}\left(\bar{x}^{n}, \bar{y}^{n}\right)\right| \\
& \quad \leqq \max \left[ \pm \exp \left( \pm 2 \xi^{\prime}(\bar{t})\right) \pm 1\right] \equiv \xi(\bar{t})
\end{aligned}
$$

and Lemma 7.1 is proved.

The next step relates to the case $\varepsilon=-$. The functional $\alpha^{0}$ figuring in (6.1) and (6.2) plays the role of an "interaction" (simple exclusion, or hard core of zero diameter). We replace it by a "cutoff" indicator $\alpha_{h}^{0}$ following the same procedure as above (see Fig. 3). We then obtain some new $\tilde{\Xi}_{\Lambda, \bar{t}}^{(3)}, \tilde{\Xi}_{\Lambda, \bar{t}}^{(3), \Omega}\left(\bar{x}^{n}, \bar{y}^{n}\right)$ and $\tilde{\varrho}_{\Lambda, \bar{t}}^{(3),}\left(\bar{x}^{n}, \bar{y}^{n}\right)$.

Lemma 7.2. There exist constants $q_{3}, c_{7}, c_{8} \in R_{+}^{1}$ depending only on $\beta$ and $\mu$ such that

$$
\left|\varrho_{\Lambda, \bar{t}}^{(3), \Omega}\left(\bar{x}^{n}, \bar{y}^{n}\right)-\varrho_{A, \bar{t}}^{(3), \Omega}\left(\bar{x}^{n}, \bar{y}^{n}\right)\right| \leqq n c_{0}^{n} c_{7}(\bar{t} \ln \bar{t}) \exp \left[-c_{8}(\ln \bar{t})^{2}\right]
$$

whenever.

We omit the proof of Lemma 7.2: it is based on arguments used for proving Lemma 6.1. We also omit the tilde and the index $\bar{t}$ from $\widetilde{\Xi}_{A, \bar{t}}^{(3)}, \Xi_{\Lambda, \bar{t}}^{(3)}, \tilde{\Xi}_{\Lambda, \bar{t}}^{(3), \Omega}\left(\bar{x}^{n}, \bar{y}^{n}\right)$, etc. The final estimate of this section takes the form (7.1) with a new function $\xi(n, \bar{t})$, $\lim _{t \rightarrow \infty} \xi(n, t)=0$ for any fixed $n=1,2, \ldots$

\section{Mixing Properties}

In this section we finish the proof of Theorem 5. The arguments used here are slight generalizations of those of [10, Section 3] and represent a variant of the transfer-matrix method. The role of the transfer-matrix is played by an integral operator acting on a functional space. The functions we consider have, as arguments, finite sets of the simple closed loops.

Denote: $\Gamma^{-}=[-h, 0], \Gamma^{+}=(0, h]$, and $\Gamma=\Gamma^{-} \cup \Gamma^{+}$where, as above, $h$ $=\operatorname{IP}\left(c_{3} \ln \bar{t}\right)+3$. Given $Y \in[\Gamma]$, a finite set of simple closed loops starting in $\Gamma$, we denote: $Y_{ \pm}=Y \cap\left[\Gamma^{ \pm}\right]$and $Y(j)=Y \cap[\Delta(j)]$ where $\Delta(j)=[j, j+1], j=-g,-h$ $+1, \ldots, h-1$. By $[\Gamma]^{\prime}$ denote the (measurable) set of elements $Y \in[\Gamma]$ which satisfy the conditions

$$
\begin{aligned}
& n(Y(j)) \leqq n_{0}, l(Y(j)) \leqq l_{0},-h \leqq j<h-1, \\
& \alpha^{0}\left(Y_{-}\right)=\alpha^{0}\left(Y_{+}\right)=1 .
\end{aligned}
$$

Let $\mathscr{L}_{2}$ denote the space $L^{2}\left([\Gamma]^{\prime}, d Y\right)$, and $\langle$,$\rangle denote the inner product in \mathscr{L}_{2}$. Let the kernel $\mathscr{K}_{h}\left(Y, Y^{\prime}\right), Y, Y^{\prime} \in[\Gamma]^{\prime}$, be given by

$$
\begin{gathered}
\mathscr{K}_{h}\left(Y, Y^{\prime}\right)=\exp \left[-U\left(T_{-h} Y_{-}\right)-U\left(T_{h} Y_{+}\right)\right. \\
\left.-U_{h}\left(\left(T_{-h} Y_{-}\right) \cup\left(T_{h} Y_{+}\right) \mid Y^{\prime}\right)+\beta \mu n\left(Y^{\prime}\right)\right],
\end{gathered}
$$

where $U_{h}(Y \mid \tilde{Y})=\sum_{\left(\omega \in Y_{\tilde{Y} \tilde{\tilde{\omega}} \in \tilde{Y}}\right.} u^{(h)}(\omega, \tilde{\omega})$ denotes the "mutual interaction energy of the loops $\omega \in Y$ and $\tilde{\omega} \in \tilde{Y}$, and $T_{t}$ denotes the space shift transformation on the vector 
$t \in R^{1}$. The exponent in (8.1) gives the difference between $U_{h}\left(\left(T_{-h} Y_{-}\right) \cup Y^{\prime} \cup\left(T_{h} Y_{+}\right)\right)$, the "total energy" of the set of loops $\left(T_{-h} Y_{-}\right) \cup Y^{\prime} \cup\left(T_{h} Y_{+}\right)$, and the energy $U\left(Y^{\prime}\right)$.

Consider the integral operator $K_{h}$ in $\mathscr{L}_{2}$ :

$$
K_{h} F(Y)=\int_{[\Gamma]^{\prime}} d Y^{\prime} \mathscr{K}_{h}\left(Y, Y^{\prime}\right) F\left(Y^{\prime}\right)
$$

It is not hard to check that $K_{h}$ is a Hilbert-Schmidt operator preserving the cone of non-negative functions $F \geqq 0$. The key remark is that $\Xi_{\Lambda}^{(3)}$ and $\Xi_{\Lambda}^{(3), \Omega}\left(\bar{x}^{n}, \bar{y}^{n}\right)$ may be written in terms of iterations of $K_{h}$.

Let $\gamma_{1}=\gamma+h-t_{2}, \gamma_{2}=\gamma-h+t_{2}$ and $\bar{\Omega}=\left[\gamma_{-}, \gamma_{+}\right]$. Consider the functions $C_{E}$, $B^{\bar{\Omega}}$ and $B^{\bar{\Omega}}\left(\cdot, \bar{x}^{n}, \bar{y}^{n}\right)$ on $[\Gamma]^{\prime}$ given by

$$
\begin{aligned}
& C_{E}(Y)= \int_{\left[\Lambda_{E}\right]} d Y_{E} \chi_{\boldsymbol{N}, \boldsymbol{L}}\left(Y_{E}\right) \alpha_{\Lambda}\left(Y_{E}\right) \alpha_{h}^{0}\left(\left(T_{\gamma_{1}} Y_{-}\right) \cup\left(T_{\gamma_{2}} Y_{+}\right) \cup Y_{E}\right) \\
& \cdot \exp \left[-U\left(Y_{E}\right)-U_{h}\left(\left(T_{\gamma_{1}} Y_{-}\right) \cup\left(T_{\gamma_{2}} Y_{+}\right) \mid Y_{E}\right)+\beta \mu n\left(Y_{E}\right)\right], \\
& B^{\Omega}(Y)= \int_{[\Omega]} d \tilde{Y} \chi_{\boldsymbol{N}, \boldsymbol{L}}(\tilde{Y}) \alpha_{h}^{0}\left(\left(T_{\gamma_{-}} Y_{-}\right) \cup\left(T_{\gamma_{+}} Y_{+}\right) \cup \tilde{Y}\right) \\
& \cdot \exp \left[-U_{h}\left(\left(T_{\gamma_{-}} Y_{-}\right) \cup\left(T_{\gamma_{+}} Y_{+}\right) \cup \tilde{Y}\right)+\beta \mu n(Y)+\beta \mu n(\tilde{Y})\right], \\
& B^{\bar{\Omega}}\left(Y ; \bar{x}^{n}, \bar{y}^{n}\right)=\frac{e^{\beta \mu n}}{n !} \underset{[\bar{\Omega} \backslash \Omega]}{f} d \tilde{Y} \underset{\left[\bar{x}^{n}, \bar{y}^{n}\right]}{f} d X \chi_{\boldsymbol{N}, \boldsymbol{L}}(Y, \tilde{Y}) \alpha_{h}^{0}\left(X,\left(T_{\gamma_{-}} Y_{-}\right) \cup\left(T_{\gamma_{+}} Y_{+}\right) \cup \tilde{Y}\right) \\
&\left.\quad \exp \left[-U_{h}\left(X,\left(T_{\gamma_{-}} Y_{-}\right) \cup\left(T_{\gamma_{+}} Y_{+}\right) \cup \tilde{Y}\right)\right]+\beta \mu n(Y)+\beta \mu n(\tilde{Y})\right] .
\end{aligned}
$$

Clearly, $C_{E}, B^{\bar{\Omega}}, B^{\bar{\Omega}}\left(\cdot ; \bar{x}^{n}, \bar{y}^{n}\right) \in \mathscr{L}_{2}$.

Lemma 8.1. The following representations hold:

$$
\begin{aligned}
& \Xi_{A}^{(3)}=\left\langle K_{h}^{\bar{t}-1} B^{\bar{\Omega}}, C_{E}\right\rangle, \\
& \Xi_{A}^{(3), \Omega}\left(\bar{x}^{n}, \bar{y}^{n}\right)=\left\langle K_{h}^{\bar{t}-1} B^{\bar{\Omega}}\left(\cdot ; \bar{x}^{n}, \bar{y}^{n}\right), C_{E}\right\rangle .
\end{aligned}
$$

Proof. (8.2) and (8.3) are verified by a direct (although quite long) calculation based on definitions introduced above. The crucial step consists of dividing the intervals $\Lambda_{I}( \pm) \backslash \bar{\Omega}$ into the intervals of the length $h$ and writing the integrand $\alpha^{0}\left(Y_{I}\right)$ $\exp \left[-U_{h}\left(Y_{I}\right)+\beta \mu n\left(Y_{I}\right)\right]$ as a product of terms corresponding to the pairs of the neighbor intervals. Such terms have the form (8.1), and it remains to use (3.6) and an obvious property of $T_{t}$-invariance.

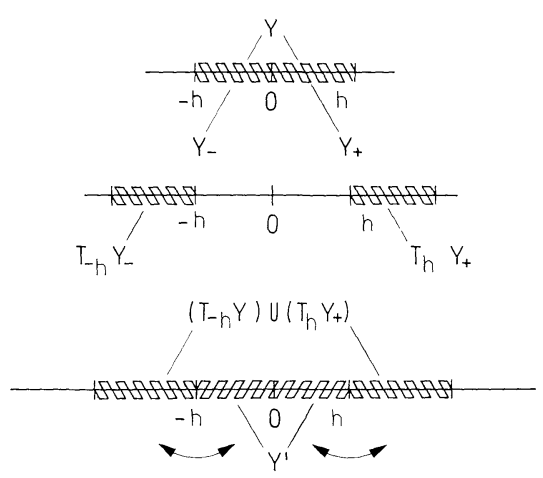

Fig. 4. The kernel $\mathscr{K}_{h}$ and the operator $K_{h}$ 
Note that in the expressions (8.2) and (8.3) only the vector $C_{E}$ depends on boundary conditions; so, only $C_{E}$ keeps the information about the finite volume $\Lambda$.

Lemma 8.2. The operator $K_{h}$ (resp., the adjoint operator $K_{h}^{*}$ ) has a unique positive eigenvector $Q_{h} \in \mathscr{L}_{2}\left(Q_{h}^{*} \in \mathscr{L}_{2}\right)$. The corresponding eigenvalue $\lambda_{h}$ is positive, nondegenerate and the largest one among the eigenvalues of $K_{h}$ (resp., $K_{h}^{*}$ ).

Proof. It is easy to check that the kernel $\mathscr{K}_{h}^{(2)}\left(Y, Y^{\prime}\right)=f d \hat{Y} \mathscr{K}_{h}(Y, \hat{Y}) \mathscr{K}_{h}\left(\hat{Y}, Y^{\prime}\right)>0$. Lemma 8.2 then follows from Proposition $\beta^{\prime \prime}$ in $[15$, p. 274].

Choose $Q_{h}$ and $Q_{h}^{*}$ so that $\left\langle Q_{h}, Q_{h}^{*}\right\rangle=1$. Denote

$$
\begin{aligned}
& \hat{K}_{h}=\lambda_{h}^{-1} K_{h} \\
& \hat{C}_{E}=\left\langle Q_{h}, C_{E}\right\rangle^{-1} C_{E} \\
& \hat{B}^{\bar{\Omega}}=\left\langle B^{\bar{\Omega}}, Q_{h}^{*}\right\rangle^{-1} B^{\bar{\Omega}} \\
& \hat{B}^{\bar{\Omega}}\left(\cdot ; \bar{x}^{n}, \bar{y}^{n}\right)=\left\langle B^{\bar{\Omega}}\left(\cdot ; \bar{x}^{n}, \bar{y}^{n}\right), Q_{h}^{*}\right\rangle^{-1} B^{\bar{\Omega}}\left(\cdot ; \bar{x}^{n}, \bar{y}^{n}\right) .
\end{aligned}
$$

According to (8.2) and (8.3),

$$
\begin{aligned}
\varrho_{\Lambda}^{(3), \Omega}\left(\bar{x}^{n}, \bar{y}^{n}\right)= & \frac{\left\langle B^{\bar{\Omega}}\left(\cdot ; \bar{x}^{n}, \bar{y}^{n}\right), Q_{h}^{*}\right\rangle}{\left\langle B^{\bar{\Omega}}, Q_{h}^{*}\right\rangle} \\
& \cdot \frac{1+\left\langle\hat{K}_{h}^{\bar{t}-1} B^{\bar{\Omega}}\left(\cdot ; \bar{x}^{n}, \bar{y}^{n}\right)-Q_{h}, \hat{C}_{E}\right\rangle}{1+\left\langle\hat{K}_{h}^{\bar{t}-1} \hat{B}^{\bar{\Omega}}-Q_{h}, \hat{C}_{E}\right\rangle} .
\end{aligned}
$$

Lemma 8.3. $\hat{C}_{E} \leqq \exp \left(4 c_{0} h\right) Q_{h}^{*}$.

We omit the proof of Lemma 8.3. The reader can reconstruct it basing on the arguments used in the proof of Lemma 3.3 in [10]. According to Lemma 8.3,

and

$$
\left|\left\langle\hat{K}_{h}^{\bar{t}-1} \hat{B}^{\bar{\Omega}}-Q_{h}, \hat{C}_{E}\right\rangle\right| \leqq \exp \left(4 c_{0} h\right)\left\langle\left|\hat{K}_{h}^{\bar{t}-1} \hat{B}^{\bar{\Omega}}-Q_{h}\right|, Q_{h}^{*}\right\rangle,
$$

$$
\left|\left\langle\hat{K}_{h}^{\bar{t}-1} \hat{B}^{\bar{\Omega}}\left(\cdot ; \bar{x}^{n}, \bar{y}^{n}\right)-Q_{h}, \hat{C}_{E}\right\rangle\right| \leqq \exp \left(4 c_{0} h\right)\left\langle\left|\hat{K}_{h}^{\bar{t}-1} \hat{B}^{\bar{\Omega}}\left(\cdot ; \bar{x}^{n}, \bar{y}^{n}\right)-Q_{h}\right|, Q_{h}^{*}\right\rangle .
$$

Lemma 8.4. Let $F \in \mathscr{L}_{2}, F \geqq 0$ and $\left\langle F, Q_{h}^{*}\right\rangle=1$. Then

$$
\left\langle\left|\hat{K}_{h}^{m} F-Q_{h}\right|, Q_{h}^{*}\right\rangle \leqq 2\left(1-\exp \left(-4 c_{0} h\right)\right)^{m-1}, \quad m=1,2, \ldots
$$

Proof. See the proof of Lemma 3.4 in [10].

Now choose the constant $c_{3}<1 / 4 c_{0}$. It is easy to check that $\exp \left(4 c_{0} h\right)$ $\left(1-\exp \left(-4 c_{0} h\right)\right)^{\bar{t}-2} \equiv \zeta(t) \rightarrow 0$ as $\bar{t} \rightarrow \infty$. Take $q_{4}$ so large that $\zeta(t)<1 / 4$ for $t>q_{4}$. Returning to (8.4), we obtain that for $\bar{t}>q_{4}$

$$
\left|\varrho_{\Lambda}^{(3), \Omega}\left(\bar{x}^{n}, \bar{y}^{n}\right)-\varrho^{(4), \Omega}\left(\bar{x}^{n}, \bar{y}^{n}\right)\right|<8 c_{0}^{n} \zeta(\bar{t}),
$$

where

$$
\varrho^{(4), \Omega}\left(\bar{x}^{n}, \bar{y}^{n}\right)=\frac{\left\langle B^{\bar{\Omega}}\left(\cdot ; \bar{x}^{n}, \bar{y}^{n}\right), Q_{h}^{*}\right\rangle}{\left\langle B^{\bar{\Omega}}, Q_{h}^{*}\right\rangle} .
$$

We used here the obvious bound: $\varrho^{(4), \Omega}\left(\bar{x}^{n}, \bar{y}^{n}\right) \leqq c_{0}{ }^{n}$. Note that the kernel $\varrho^{(4), \Omega}$ does not depend on $\Lambda$.

Summarizing the estimates of Sections 5-8, we get:

$$
\left|\varrho^{(4), \Omega}\left(\bar{x}^{n}, \bar{y}^{n}\right)-\varrho_{\Lambda}^{(\Omega)}\left(\bar{x}^{n}, \bar{y}^{n}\right)\right|<\delta\left(n, t_{1}, \bar{t}\right),
$$


where $\lim \delta\left(n, t_{1}, \bar{t}\right)=0$ as $t_{1}, \bar{t} \rightarrow \infty$ for any fixed $n=1,2, \ldots$ Bound (8.6) holds whenever (5.1) holds. Since $\varrho^{(4), \Omega}\left(\bar{x}^{n}, \bar{y}^{n}\right)$ does not depend on $A$, bound (8.6) proves Theorem 5 .

Concluding Remarks. 1 . The condition $\Phi \geqq 0$ has been repeatedly used in technicalities in the course of the proof of Theorem 5. However, the only points where it is essential are Lemmas 8.3 and 8.4.

2. The restrictive condition a) of the fast decreasing imposed on $\Phi$ plays the critical role for our choice of $n_{0}, l_{0}$ and $h$. The relations between $n_{0}, l_{0}, h$, and $\bar{t}$ are dicted by the cruicial bound (8.5) of Lemma 8.4 .

Acknowledgements. The author thanks Professors R. Stora. M. Mebkhout, D. Ruelle, and N H. Kuiper for the warm hospitality at CPT CNRS and UER de Lumini, Marseille, and I.H E.S. Bures-sur-Yvette where the work has been partially performed. It also gives the pleasure to thank the Mathematical Institute of Camerino University for kind hospitality.

\section{Appendix : Some Generalizations}

The methods of this paper may be also applied to the case of one-dimensional c.q.s. consisting of a finite number of species. In that case, instead of the Fock spaces $\mathscr{H}_{\varepsilon}(\Omega)$ associated to a b.B.s. $\Omega$ we take the tensor product $\mathscr{H}_{m_{+}, m_{-}}(\Omega)$ $=\left(\mathscr{H}_{+}(\Omega)^{\otimes m_{+}}\right) \otimes\left(\mathscr{H}_{-}(\Omega)^{\otimes m_{-}}\right)$where $m_{+}, m_{-}$are fixed non-negative integers giving the number of boson and fermion species. Let $\mathfrak{A}_{\Omega}^{m_{+}, m_{-}}$be the $c^{*}$-algebra of bounded operators in $\mathscr{H}_{m_{+}, m_{-}}(\Omega)$ and $\mathfrak{A}^{m_{+}, m_{-}}$the $B^{*}$-algebra of the corresponding c.q.s. in $R^{1}$.

Fix a $\left(m_{+}+m_{-}\right)$-vector $\boldsymbol{n}$ consisting of non-negative integers $\left(n_{+}^{(1)}, \ldots, n_{+}^{\left(m_{+}\right)}, n_{-}^{(1)}, \ldots, n_{-}^{\left(m_{-}\right)}\right)$. The $\boldsymbol{n}$-particle Hamiltonian $H_{m_{+}, m_{-}}(\Lambda)$ in a bounded interval $\Lambda$ is defined as $P_{m_{+}, m_{-} ; \boldsymbol{n}} H_{\boldsymbol{n}} P_{m_{+}, m_{-} ; \boldsymbol{n}}$ where

$$
P_{m_{+}, m_{-;}}: L^{2}\left(\Lambda^{|\boldsymbol{n}|}\right) \rightarrow\left(\bigotimes_{i=1}^{m_{+}} L_{+}^{2}\left(\Lambda^{n_{+}(i)}\right)\right) \otimes\left(\bigotimes_{i=1}^{m-} L^{2}\left(\Lambda^{n(i)}\right)\right)
$$

is the "canonical" projection, $|\boldsymbol{n}|=\sum_{i=1}^{m_{+}} n_{+}^{(i)}+\sum_{i=1}^{m_{-}} n_{-}^{(i)}$ and $H_{\boldsymbol{n}}$ is given by $(0.1)$, possibly replacing the potential $\Phi$ by a family of non-negative pair potentials $\Phi_{i_{\varepsilon}, i_{\varepsilon}{ }^{\prime}}$ describing the interaction of particles belonging to the species $i_{\varepsilon}$ and $i_{\varepsilon^{\prime}}^{\prime}, i_{\varepsilon}=1, \ldots, m_{\varepsilon}$, $i_{\varepsilon^{\prime}}^{\prime}=1, \ldots, m_{\varepsilon^{\prime}}, \varepsilon, \varepsilon^{\prime}= \pm$. We impose the condition that $\Phi_{i_{+}, i_{+}}$(boson "self-potential") satisfies conditions a) and b), $i_{+}=1, \ldots, m_{+}$, and all other $\Phi^{\prime} s$ satisfy condition a).

Fix $\beta>0$ and a $\left(m_{+}+m_{-}\right)$-vector $\bar{\mu}$ consisting of reals $\left(\mu_{+}^{(1)}, \ldots, \mu_{+}^{\left(m_{+}\right)}, \mu_{-}^{(1)}, \ldots, \mu_{-}^{\left(m_{-}\right)}\right)$. The Gibbs state $G_{\beta, \mu_{,}, m_{-}}^{m_{+}}$is given by the formula similar to (1.1). As in Section 1, we introduce the operators $g_{\beta, \bar{\mu}, m_{-}}^{m_{+}, \Omega}$ and their kernels $\varrho_{\beta, \bar{\mu}, \Lambda}^{m_{+}, m_{-} ; \Omega}, \Omega \subseteq \Lambda$.

Theorem 7. Let $A \in \mathfrak{Q}^{m_{+}, m_{-}}$. For any $\beta \in R_{+}^{1}$ and $\bar{\mu} \in R^{m_{+}+m_{-}}$there exists the limit

$$
G_{\beta, \bar{\mu}}^{m_{+}, m_{-}}(A)=\lim _{\Lambda \rightarrow R^{1}} G_{\beta, \bar{\mu}, A}^{m_{+}, m_{-}}(A) .
$$

The limit state $G_{\beta, \bar{\mu}}^{m_{+}, m_{-}}$is a locally normal and extremal invariant state under the action of the space translation group $\left\{\tau_{a}, a \in R^{1}\right\}$.

As for the case of a c.q.s. consisting of identical particles, this assertion is recuced to statements about the convergence of the kernels $\varrho_{\beta, \mu, m_{-}}^{m_{+}, \Omega}$ and properties of the limit kernels $\varrho_{\beta, \bar{\mu}}^{m_{+}, m_{-} ; \Omega}$. These statements are based on the Wiener 
integral representation. Instead of the ensemble of Wiener trajectories interacting via fixed $\Phi$, we now have the ensemble of trajectories of various kinds. The key remark is that all trajectories are simple (of the length $\beta$ ) due to condition b) for $\Phi_{i_{+}, i_{+}}$. The further analysis develops following the same scheme and we omit it.

The next step is to introduce several types of particles inside the fixed species. Here we also can consider various interaction potentials and vector-valued chemical potentials. We left to the reader the formulation of the corresponding theorem.

The case of the Boltzmann statistics is also covered by our methods. For this case a natural object characterizing the infinite system is the reduced density matrices [7]. Their representation in terms of the Wiener integral and its analysis do not differ from those given in Sections 3 and 5-8. As the result, we get the existence of the limit RDM and their cluster property.

\section{References}

1. Ruelle,D.: Statistical mechanics. Rigorous results. New York and Amsterdam: W. A. Benjamin, Inc. 1969

2. Emch, G. : Algebraic methods in statistical mechanics and quantum field theory. New York: WileyInterscience 1972

3. Dubin,D. A. : Solvable models in algebraic statistical mechanics. Oxford: Clarendon Press 1974

4. Araki,H.: Uniqueness of KMS states of one-dimensional quantum lattice systems. Commun. math. Phys. 44, 1-7 (1975)

5. Dyson, F.J., Lieb, E.H., Simon, B. : Phase transitions in the quantum Heisenberg model. Phys. Rev. Letters 37, 120-122 (1977); J. Stat. Phys. (to appear) (1978)

6. Lewis.J.T., Pulé,J.V.: The free boson gas in a rotating bucket. Commun. math. Phys. 45, 115 131 (1975)

7. Ginibre,J.: Some applications of functional integration in statistical mechanics. In: Statistical mechanics and quantum field theory (Les Houches Summer School of Theoretical Physics) (eds. C. DeWitt ,R. Stora). New York-London-Paris: Gordon and Breach 1970

8a.Souhov, Iu. M. : Sur les systèmes continus de la Méchanique statistique quantique a une dimension avec une intération de portée infinie: Compt. Rend. A.S. Paris, Sér. A 279, 433-434 (1974)

8b.Souhov,Iu.M.: Absence de transitions de phases dans les systèmes continus de la Mécanique statistique quantique a une dimension. Compt. Rend. A.S. Paris, Sér. A 279, $475-477$ (1974)

9. Robinson, D.W.: Bose-Einstein condensation with attractive boundary conditions. Commun. math. Phys. 50, 53-60 (1976)

10. Suhov, Yu. M.: Random point processes and DLR equations. Commun. math. Phys. 50, 113-132 (1976)

11. Suhov, Yu.M.: Existence and regularity of the limit Gibbs state for one-dimensional continuous systems of quantum statistical mechanics. Soviet Math. (Dokl.) 11 (195), 1629-1632 (1970)

12. Gikhman,I.I., Skorokhod,A.V.: Theory of random processes, Vol. II (In Russian). Moscow: Nauka 1973

13. Gikhman,I.I., Skorokhod,A.V.: Introduction into theory of random processes (Russian). Moscow: Nauka 1965

14. Suhov, Yu.M.: Limit density matrices for one-dimensional continuous systems of quantum statistical mechanics (Russian). Matem. Sbornik 83, 491--512 (1970)

15. Krein,M.G., Rutman,M.A.: Linear operators leaving invariant a cone in a Banach space. In: Functional analysis and measure theory. AMS Transl. Ser. 1, Vol. 10, pp. 199-325. Providence, RI: American Mathematical Society 1962

Communicated by E. Lieb

Received April 25, 1977; in revised form December 16, 1977 